\title{
Anormalidades dentárias em Procyon cancrivorus (Carnivora, Procyonidae): Relato de caso
}

\author{
Dental abnormalities in Procyon cancrivorus \\ (Carnivora, Procyonidae): Case report
}

Renato Zacarias Silva ${ }^{[a]}$, Fernando Marques Quintela ${ }^{[b]}$

\section{Resumo}

A ocorrência de dentes pré-molares superiores supranumerários e de má-oclusão dental, assim como os efeitos dessas anormalidades no sistema mastigatório são registrados em Procyon cancrivorus. 0 sincrânio de um macho de $P$. cancrivorus, morto por atropelamento, apresenta anormalidades de posicionamento e no número dentário dos pré-molares superiores e odontopatologias estruturais. 0 quarto pré-molar direito e esquerdo superior são duplicados gerando apinhamento dentário com deslocamento buco-lingual, má-oclusão dentária, diminuição da superfície palatina, comprometimento do aparelho carniceiro (dentes carnassiais), atrição dos pré-molares inferiores, fratura cuspedal e exposição da câmara pulpar. Não há braquignatia, prognatia, anormalidades na junção têmporo-mandibular ou cáries. A formação extranumerária dos botões dos dentes permanentes durante o desenvolvimento da lâmina dental sucessional pode ter acarretado a anormalidade numérica e funcional que sobrecarregou os molares direitos durante os processos de mastigação.

Palavras-chave: Procyonidae. Dentes supranumerários. Má-oclusão dentária.

\begin{abstract}
The occurrence of supernumerary upper premolar teeth, dental malocclusion and its effects on the masticatory system has been found in Procyon cancrivorus. The sincranium of a male P. cancrivorus, killed by overrunning, shows abnormalities in premolar teeth number and position and structural odontopathologies. The right and left superior fourth premolars are duplicated causing fluff-lingual displacement, dental malocclusion, reduced palatal surface, damage to the butcher device (carnassials teeth) and attrition of premolar teeth, cuspedal fracture and exposure of the pulp chamber. There are no brachygnathism, prognathism, abnormalities in the temporomandibular joint or caries. The formation of supernumerary buds of permanent teeth during the development of the successional dental lamina may have caused the numeric and functional abnormalities that overloaded the right molars during the masticatory process in the specimen.
\end{abstract}

Keywords: Procyonidae. Supernumerary teeth. Dental Malocclusion.

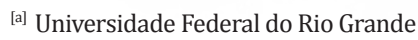
(FURG), Instituto de Ciências Biológicas (ICB), Laboratório de Morfologia Funcional, Rio Grande, RS - Brasil, e-mail: renatinhoterciotti@yahoo.com.br

[b] Universidade Federal do Rio Grande do Sul, Programa de Pós-graduação em Biologia Animal, Porto Alegre, RS - Brasil.
Recebido: 22/02/2010

Received: 02/22/2010

Aprovado: 18/10/2010

Approved: 10/18/2010 
A saúde oral se relaciona com a sistêmica e confere à odontologia veterinária valor crescente (1); todavia, para animais selvagens, a clínica médica odontológica preventiva é tardia, pouco compreendida e pouco documentada pelos pesquisadores (2). Anormalidades de posicionamento dentário podem ser resultantes da conformação mandibular ou, mais raramente, de padrões de erupção anormais espontâneos e dentes supranumerários (3).

Um exemplar macho adulto de Procyon cancrivorus Cuvier, 1798 foi encontrado morto por atropelamento em 25 de julho de 2007 na rodovia BR 471 ( $\left.32^{\circ} 06.054^{\prime} \mathrm{S} ; 52^{\circ} 19.664^{\prime} \mathrm{W}\right)$, Rio Grande, RS, próximo a um canal de escoamento adjacente a uma área aberta com predomínio de vegetação gramínea e arbustiva. 0 espécimen foi mensurado e preparado para osteologia de acordo com Auricchio e Salomão (4). As medidas do sincrânio seguem Rosas (5) e Schiller (6), com adaptações. 0 sincrânio foi enviado para a coleção da
Fundação Zoobotânica do Rio Grande do Sul (FZBRS) para registro (número de referência MCN 3072). A Tabela 1 apresenta as medidas e os valores morfométricos corporais e sincraniais mensurados do exemplar.

O sincrânio apresenta anormalidades no posicionamento, no número de pré-molares superiores e alterações dentárias estruturais (Figuras 1A e 1B). 0 quarto par de pré-molares superiores é duplicado, conferindo ao exemplar a fórmula dentária (FD): 3/3 1/1 5/4 2/2 e gerando apinhamento dentário que forçou os dentes duplicados a se deslocarem bucolingualmente (lado a lado). Este reposicionamento dentário acarretou má-oclusão e diminuição da superfície palatal. A oclusão normal é uma relação dentária harmoniosa e a má-oclusão constitui uma anomalia do desenvolvimento dentário e/ou dos arcos dentários, ocasionando problemas estético-funcionais (7) (Figura 1B). Em P. cancrivorus a FD normal é: 3/3 1/1 4/4 e 2/2 (8).

Tabela 1 - Medidas corporais e sincraniais para o espécimen de Procyon cancrivorus

\begin{tabular}{|c|c|}
\hline Medidas Corporais & Valor $(\mathrm{cm})$ \\
\hline Comprimento total & 97 \\
\hline Comprimento cabeça e corpo & 65 \\
\hline Medidas do Sincrânio & Valor $(\mathrm{cm})$ \\
\hline Comprimento condilobasal & 11,86 \\
\hline Comprimento basilar de Hensel & 10,9 \\
\hline Comprimento da série pós-canina superior & 3,81 \\
\hline Comprimento hâmulo pterigoideo - osso incisivo (pré-maxilar) & 8,2 \\
\hline Largura zigomática & 8,36 \\
\hline Largura rostral & 2,92 \\
\hline Largura mastoide & 6,83 \\
\hline Altura do crânio (basisfenoide - inserção do processo interparietal) & 4,27 \\
\hline Comprimento do palato & 6,85 \\
\hline Largura do palato no pré-molar 1 & 1,91 \\
\hline Largura do palato no pré-molar 4 & 1,4 \\
\hline Largura do palato no molar 2 & 2,41 \\
\hline Largura máxima do côndilo occipital & 2,75 \\
\hline Comprimento máximo dos nasais & 3,33 \\
\hline Largura da constrição interorbital & 2,75 \\
\hline Largura do processo supraorbital & 3,34 \\
\hline Largura máxima interna do orifício nasal & 1,33 \\
\hline Comprimento da mandíbula & 8,74 \\
\hline Largura máxima do processo coronoide & 1,27 \\
\hline Altura da mandíbula até o processo coronoide & 5,06 \\
\hline Largura da mandíbula & 0,95 \\
\hline
\end{tabular}

Fonte: Dados da pesquisa. 

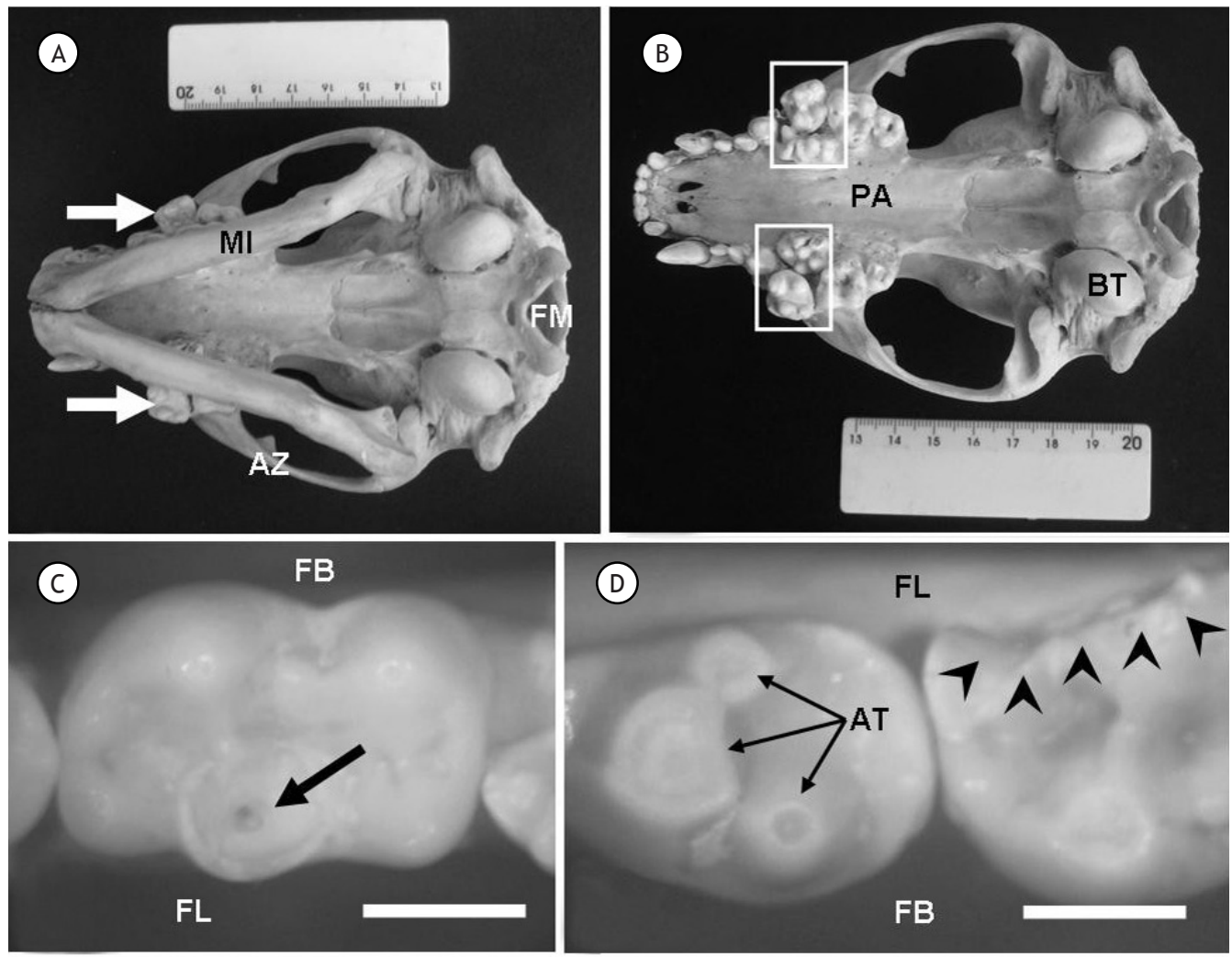

(D)

FL
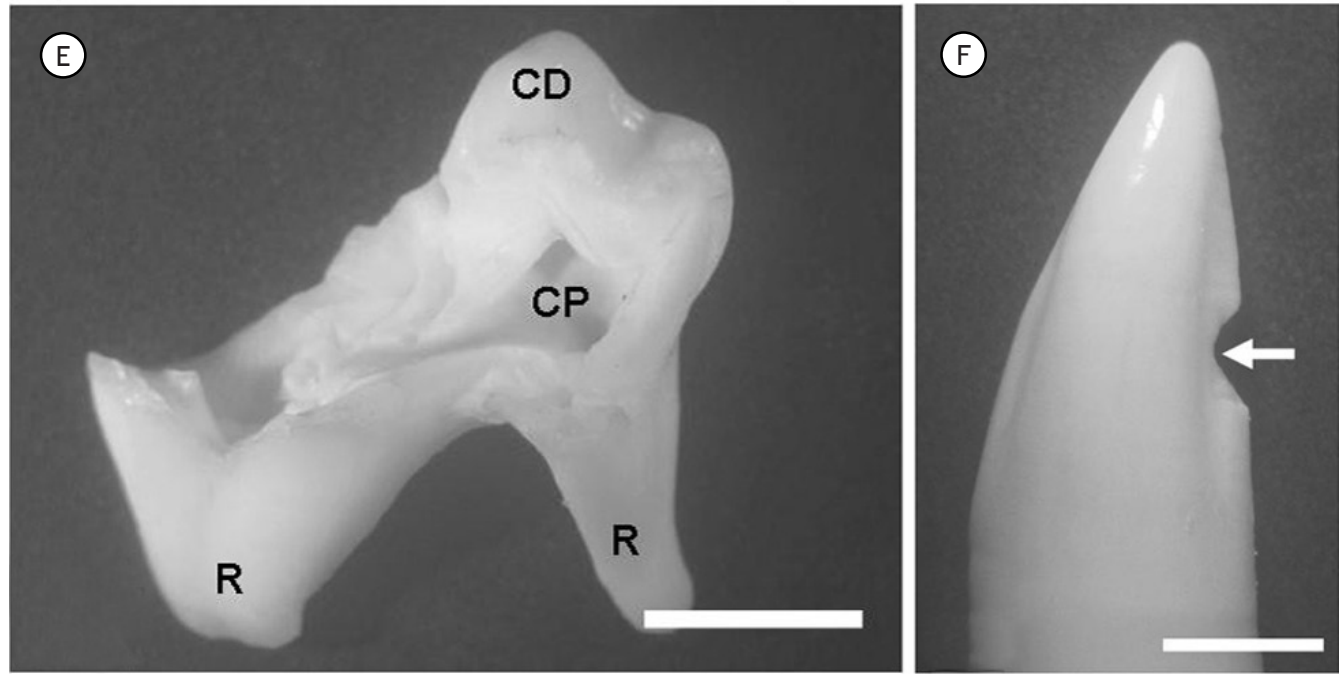

Figura 1 - Procyon cancrivorus. A: Sincrânio em vista ventral. Observar o posicionamento anormal (mal ocluso) dos quartos pré-molares superiores (setas) com relação ao alinhamento da mandíbula (MI). FM - Forâmen magno; AZ - Arco zigomático. Escala: centimétrica. B: Crânio em vista ventral. Atentar para a duplicação dos quartos pré-molares superiores (quadros) e a redução da área do palato (PA). BT - bula timpânica. Escala: centimétrica. C: Primeiro molar inferior direito. Notar a fratura da cúspide com exposição da câmara pulpar (seta). FL - Face lingual; FB - Face bucal. Escala: 0,4 cm. D: Quarto pré-molar inferior esquerdo com áreas de atrição (AT) e primeiro molar inferior esquerdo com perda de tecido dentário por fratura vertical na face lingual da coroa (cabeças de seta). FL - Face lingual; FB - Face bucal. Escala: 0,4 cm. E: Vista da face bucal do segundo molar inferior direito. Observar a extensa degeneração da estrutura dental com ampla exposição da câmara pulpar (CP) e remanescente de uma cúspide 
No espécimen de P. cancrivorus, a má-oclusão comprometia o funcionamento do característico aparelho carniceiro (AC, dentes carnassiais) dos Carnívora (9) e os molares direitos inferiores podem ter sido mais exigidos durante a mastigação, em um processo compensatório da falha do AC. Os pré-molares inferiores apresentam pequena atrição na coroa dental. Os primeiros molares inferiores direito e esquerdo apresentam fratura das cúspides com exposição da câmara pulpar. $\mathrm{O}$ segundo molar inferior direito apresenta a coroa dentária quase totalmente destruída, com uma pequena porção lingual presente. 0 canino superior direito mostra fratura de esmalte na face distal, e o canino inferior esquerdo apresenta fratura horizontal da coroa (Figuras $1 \mathrm{~A}$ a $1 \mathrm{~F}$ ). Desgastes dentários e fraturas são registrados por Braunn e Ferigolo (10) em Otaria flavescens ( $=0$. byronia; Pinnipedia) e por Aguiar et al. (11) em Didelphis albiventris (Didelphimorpha). Alterações rostrais severas que podem conduzir a alterações dentárias foram registradas em Pontoporia blainvillei (Pontoporiidae) por Gerholdt (12), todavia a autora não analisa efeitos da má-oclusão. 0 espécimen de $P$. cancrivorus não apresentou braquignatia, prognatia, anormalidades na articulação temporomandibular e cáries. Durante a formação da lâmina dental sucessional, os botões dos quartos pré-molares superiores podem ter sido gerados supranumerariamente, como registra Avery (13) para a espécie humana.

\section{Referências}

1. Gioso MA. Afecções periodontais. Cadernos Técnicos da UFMG. 1997;20:71-80.

2. Robinson PT. Oral pathology in mammals at San Diego Zoo and Wild Animal Park. Proc Am Assoc Zoo Vet. 1979; p. 96-98.

3. Van Kruiningen, HJ. Sistema gastrointestinal. In: Carlton WW, McGavin MD. Patologia veterinária especial de Thomson. Porto Alegre: Artes Médicas Sul Ltda; 1998. p. 13-94.
4. Auricchio P. Esqueletos. In: Auricchio P, Salomão MG. (Org.). Técnicas de coleta e preparação de vertebrados para fins científicos e didáticos. Arujá: Instituto Pau Brasil de História Natural; 2002. p. 195-216.

5. Rosas FCW. Aspectos da dinâmica populacional e interações com a pesca do leão marinho do sul, Otaria flavescens (Shaw, 1800) (Pinnipedia, Otariidae) no litoral sul do Rio Grande do Sul, Brasil [dissertação]. Rio Grande: Fundação Universidade Federal do Rio Grande; 1989.

6. Schiller DMMM. Idade e desenvolvimento craniano do lobo marinho do sul Arctocephalus australis (Zimmermann, 1783) (Carnivora: Otariidae), no litoral do Rio Grande do Sul, Brasil [dissertação]. Rio Grande: Fundação Universidade Federal do Rio Grande; 2000.

7. Thomaz EBAF, Valença MG. Prevalência de má-oclusão e fatores relacionados à sua ocorrência em pré-escolares da cidade de São Luís - MA - Brasil. RPG Rev Pos Grad. 2005;12(2):212-21.

8. Parera A. Los mamíferos de la Argentina y la región austral de Sudamérica. Buenos Aires: El Ateneo; 2002. p. 192-4.

9. Pough FH, Heiser JB, McFarland WN. A vida dos vertebrados. São Paulo: Atheneu 1993. p. 677-739.

10. Braunn PR, Ferigolo J. Osteopatologias e alterações dentárias em Otaria byronia (Pinnipedia, Otariidae) da costa do Rio Grande do Sul, Brasil. Iheringia, Série Zoológica. 2005;94(2):117-22.

11. Aguiar MS, Ferigolo J, Rossi JL Jr., Gioso MA. Atrição dental em Didelphis albiventris e D. marsupialis (Marsupialia, Didelphimorphia, Didelphidae) do sul do Brasil. Ciência Rural. 2004;34(4):1127-32.

12. Gerholdt JM. Abnormalities and pathologies in the scout of La Plata Dolphin (Pontoporia blainvillei). The Ectophora: The Newsletter of the Calvert Marine $\mathrm{Mu}$ seum Fossil Club. 2006;21(3):1-5.

13. Piesco, NP, Avery, JK. Development of teeth: crown formation. In: Avery JK. Oral development and histology. New York (U.S.A): Thieme Medical Publishers; 2002. p. 72-107. 